\title{
Correction to: Strong convergence of a linearization method for semi-linear elliptic equations with variable scaled production
}

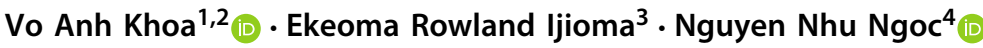

Published online: 8 January 2021

(c) SBMAC - Sociedade Brasileira de Matemática Aplicada e Computacional 2021

\section{Correction to: Computational and Applied Mathematics (2020) 39:281 https://doi.org/10.1007/s40314-020-01334-0}

In Section 1.2 of the original publication Khoa et al. (2020b), we stated that

"Cf. Khoa and Muntean (2016) as our initiation, a linearization scheme was briefly designed to prove the weak solvability of $\left(P_{\varepsilon}\right)$ as $\alpha=0$. However, this result was only guaranteed when the diffusion must be very larger than the Lipschitz rate of reactions. Our next evolution in this area went to the work Khoa et al. (2020a) where, for the first time, we addressed a linearization scheme for the weak solvability of a semi-linear microscopic system with real variable scalings."

This statement can be misunderstood to the fact that the scheme is newly designed in Khoa et al. (2020b), based on Khoa and Muntean (2016). Similarly, in the abstract of the original publication Khoa et al. (2020b), we stated that

"This work is devoted to the development and analysis of a linearization algorithm for microscopic elliptic equations, with scaled degenerate production, posed in a perforated medium and constrained by the homogeneous Neumann-Dirichlet boundary conditions."

The original article can be found online at https://doi.org/10.1007/s40314-020-01334-0.

Nguyen Nhu Ngoc

nhungoc.nguyen@polimi.it

Vo Anh Khoa

vakhoa.hcmus@gmail.com; anhkhoa.vo@uncc.edu

Ekeoma Rowland Ijioma

e.rijioma@gmail.com

1 Department of Mathematics and Statistics, University of North Carolina at Charlotte, Charlotte, NC 28223, USA

2 Faculty of Sciences, Hasselt University, Campus Diepenbeek, Agoralaan Building D, BE3590 Diepenbeek, Belgium

3 Meiji Institute for Advanced Study of Mathematical Sciences, 4-21-1 Nakano, Nakano-ku, Tokyo, Japan

4 Dipartimento di Matematica, Politecnico di Milano, 20133 Milan, Italy 
It is likely that we develop a new scheme in the paper. Those two statements, however, are not what we meant for in the paper. Therefore, in the present corrigendum, we want to correct this point by adding proper references and clarifications.

In our publication Khoa et al. (2020b), we mainly got inspired from the works Slodička (2001) and Mitra and Pop (2019). However, the original version of this linearization scheme was proposed in Pop and Yong (2000), where the authors constructed an $H^{1}$-fixed-point regularization scheme to prove the existence and uniqueness results for a quasilinear elliptic equation with non-Lipschitz reaction. The approach we have proposed in Section 3 of Khoa et al. (2020b) and particularly, our main Theorems 2 and 3 obtained therein can also be found in that paper. It can be seen that our Theorems 2 and 3 were obtained based on the same $H^{1}$-type contraction argument.

It is clear that for nonlinear parabolic problems and their related time-dependent systems, having the Euler-implicit scheme usually leads to nonlinear elliptic-like problems. That naturally requires a linearization scheme for the sake of numerical analysis. Therefore, many nonlinear time-dependent problems in the literature were solved in line with this procedure. In particular, a quite early application of linearization schemes is found in Pop et al. (2004), where a class of degenerate parabolic problems including the Richards' equation was solved using that scheme. It turns out that after regularization for the nonlinear term and using an Euler implicit discretization in time, the emerging nonlinear elliptic problems were obtained with Lipschitz reactions. Then, analysis of a linearization scheme was investigated. We also provide here the work Kumar et al. (2013), where the authors used the so-called $L$ linearization scheme to prove the existence of a solution to a dissolution-precipitation model. In the same vein, some extensions gained the applicability of the linearization procedure to cope with a two-phase model in Radu et al. (2015) and with a tri-phase one in Redeker et al. (2016). Later on, the authors in Radu et al. (2017) proposed a new type of the $L$-scheme for Hölder-type nonlinearities without using regularization. Note that the $L$-scheme with regularization is exactly what we have done in Khoa et al. (2020b) when proving the existence and uniqueness of the microscopic solution.

\section{References}

Khoa VA, Muntean A (2016) Asymptotic analysis of a semi-linear elliptic system in perforated domains: well-posedness and correctors for the homogenization limit. J Math Anal Appl 439(1):271-295. https:// doi.org/10.1016/j.jmaa.2016.02.068

Khoa VA, Thieu TKT, Ijioma ER (2020a) On a pore-scale stationary diffusion equation: scaling effects and correctors for the homogenization limit. Discrete Contin Dyn Syst B. https://doi.org/10.3934/dcdsb. 2020190

Khoa VA, Ijioma ER, Ngoc NN (2020b) Strong convergence of a linearization method for semi-linear elliptic equations with variable scaled production. Comput Appl Math. https://doi.org/10.1007/s40314-02001334-0

Kumar K, Pop IS, Radu FA (2013) Convergence analysis for a conformal discretization of a model for precipitation and dissolution in porous media. Numerische Mathematik 127(4):715-749. https://doi.org/ 10.1007/s00211-013-0601-1

Mitra K, Pop IS (2019) A modified L-scheme to solve nonlinear diffusion problems. Comput Math Appl 77(6): 1722-1738. https://doi.org/10.1016/j.camwa.2018.09.042

Pop IS, Yong WA (2000) On the existence and uniqueness of a solution for an elliptic problem. Studia Universitatis Babe Bolyai Mathematica 45:97-107

Pop IS, Radu F, Knabner P (2004) Mixed finite elements for the Richards' equation: linearization procedure. J Comput Appl Math 168(1-2):365-373. https://doi.org/10.1016/j.cam.2003.04.008

Radu FA, Nordbotten JM, Pop IS, Kumar K (2015) A robust linearization scheme for finite volume based discretizations for simulation of two-phase flow in porous media. J Comput Appl Math 289:134-141. https://doi.org/10.1016/j.cam.2015.02.051 
Radu FA, Kumar K, Nordbotten JM, Pop IS (2017) A robust, mass conservative scheme for two-phase flow in porous media including Hölder continuous nonlinearities. IMA J Numer Anal 38(2):884-920. https:// doi.org/10.1093/imanum/drx032

Redeker M, Rohde C, Pop IS (2016) Upscaling of a tri-phase phase-field model for precipitation in porous media. IMA J Appl Math 81(5):898-939. https://doi.org/10.1093/imamat/hxw023

Slodička M (2001) Error estimates of an efficient linearization scheme for a nonlinear elliptic problem with a nonlocal boundary condition. ESAIM Math Model Numer Anal 35(4):691-711. https://doi.org/10.1051/ m2an:2001132

Publisher's Note Springer Nature remains neutral with regard to jurisdictional claims in published maps and institutional affiliations. 